\title{
A generic temperature model for solar photobioreactors
}

\author{
V. Goetz ${ }^{\mathrm{a}, *}$, F. Le Borgne ${ }^{\mathrm{b}}$, J. Pruvost ${ }^{\mathrm{b}}$, G. Plantard ${ }^{\mathrm{a}}$, J. Legrand ${ }^{\mathrm{b}}$ \\ a PROMES-CNRS, UPR 8521, Rambla de la Thermodynamique, 66100 Perpignan, France \\ b GEPEA, Université de Nantes, CNRS, UMR 6144, boulevard de l'Université, CRTT-BP406, 44602 Saint-Nazaire Cedex, France
}

\begin{abstract}
A B S T R A C T
With the objective of addressing issues related to the temperature control of a solar closed photobioreactor (PBR), a laboratory set-up representative of an intensified closed flat panel PBR was designed. Only its thermal behaviour was considered, with no cultivation of microalgae. It was characterized indoors under controlled artificial irradiation and outdoors under solar irradiation on sunny days in summer. A simplified thermal model to predict the temperature time course was developed and validated by comparison with experimental results. The thermal model was then used as a simulation tool to investigate relevant parameters influencing thermal behaviour, such as the optical properties of the illuminated surface and the surrounding wind speed. A heat balance was applied for different cases to determine the energy consumption during one year of operation with temperature regulated in a range suitable for microalgae growth.
\end{abstract}

\section{Introduction}

Solar cultivation of photosynthetic microorganisms (microalgae and cyanobacteria) is considered as a promising process for the production of primary resources [1-7]. As in any light-driven process, the amount of light supplied to the culture is one of the most important parameters influencing productivity [8-13]. Optimization of the productivity of the cultivation systems has led to the design and development of photobioreactors (PBRs) presenting very large illuminated surface areas relative to the volume of the bulk culture $[14,15]$. Unlike other systems such as open ponds, also used for microalgal cultivation, PBRs have closed geometries. This feature, combined with a high ratio of illuminated surface area to culture volume, leads to a very low thermal inertia. As a consequence solar PBRs have a design highly favorable to marked temperature changes in the culture [11] with a significant tendency to overheating, especially in summer due to the strong irradiation and the high ambient temperature. Microorganisms are very sensitive to temperature [16-19], with optimal values usually in the range $288-308 \mathrm{~K}$, depending on the species. High temperatures likely to be reached at solar noon on sunny days may cause partial deterioration or even destruction of the culture. Temperature control is thus crucial in an outdoor PBR. Various solutions have been suggested in the open literature. They are based on water cooling, including direct immersion of the PBR in a thermo-regulated pool $[20,21]$ or water spraying on the PBR surface [22-24]. Such procedures are efficient, but incur a significant additional operating cost. If the aim is to produce biofuel, a strong need for cooling will also have a negative impact on the energy balance, and so the utility of the PBR technology, i.e. high productivity, can be negated.

An exact understanding of thermal impact on PBR productivity requires theoretical and engineering development and outdoor experiments. Because of the time variability of both, solar radiation level and ambient temperature, such experiments should be conducted over at least a full year of exploitation. Meanwhile, before undertaking this necessary and comprehensive research work, useful information can be obtained with temperature profiles for given, well-chosen year periods (summer, for example). Such information will, in particular, allow an assessment of whether the control of the temperature should be considered as a major issue in solar closed PBRs, especially in the case of biofuels production, where energy consumption is a key criterion.

The generic model developed by Pruvost et al. [10] in a recent paper permits to quantify the high dependence of PBR operation and productivities on solar radiation conditions. This model was adapted to the case of a PBR in which light alone limits growth. Parameters like $\mathrm{pH}$, inorganic dissolved carbon, minerals nutrients and temperature, were kept at their optimal values. Beyond the thermal experiments carried out to estimate the temperature range that have to be supported by a culture in a closed solar PBR, the work described here addresses the development of a thermal model that, if necessary, could be coupled with the light-limited model.

For this study, the simplest PBR design, corresponding to a horizontal flat panel was retained. If needed, the approach could easily be extended and adapted to any other designs such as vertical or tilted panels, tubular or cylindrical PBRs. In a first step, a laboratory 


\begin{tabular}{|ll|}
\hline \multicolumn{2}{|l|}{ Nomenclature } \\
$C p$ & heat capacity $\left(\mathrm{J} \mathrm{kg}^{-1} \mathrm{~K}^{-1}\right)$ \\
$h$ & convective heat exchange coefficient $\left(\mathrm{W} \mathrm{m}^{-2} \mathrm{~K}^{-1}\right)$ \\
$m$ & mass $(\mathrm{kg})$ \\
$q$ & total solar radiation $\left(\mathrm{W} \mathrm{m}^{-2}\right)$ \\
$S$ & surface area $\left(\mathrm{m}^{2}\right)$ \\
$T$ & temperature $(\mathrm{K})$ \\
$v$ & wind speed $\left(\mathrm{m} \mathrm{s}^{-1}\right)$ \\
& \\
Greek letters \\
$\alpha$ & absorptivity $(-)$ \\
$\varepsilon$ & emissivity $(-)$ \\
$\rho$ & reflectivity $(-)$ \\
$\sigma$ & Boltzman constant $\left(\mathrm{W} \mathrm{m}^{-2} \mathrm{~K}^{-4}\right)$ \\
$\tau$ & transmissivity $(-)$ \\
Subscripts \\
amb & ambient \\
$\mathrm{g}$ & glass \\
$\mathrm{s}$ & solution/culture \\
$\mathrm{u}$ & underside of the reactor \\
\end{tabular}

set-up representative of an intensified closed PBR was designed (flat panel geometry with a high specific illuminated surface area). Its thermal behaviour was characterized under both controlled artificial irradiation and natural solar irradiation on a sunny day in summer. Only thermal behaviour was considered, with no cultivation of microalgae. In a second step, a thermal model to predict culture temperature time course was developed and the simulated results were compared with the experimental ones. Finally, the thermal model was used to simulate temperature during one year of operation, and to study relevant parameters influencing thermal behaviour, such as optical properties of the illuminated surface and surrounding wind speed. A heat balance was applied for different cases to determine energy consumption with temperature regulated in a range suitable for microalgae growth.

\section{Experimental set-up}

The closed PBR (Fig. 1) was a flat panel type with an illuminated surface area of $0.33 \mathrm{~m}^{2}$ and a total volume of $4.5 \times 10^{-3} \mathrm{~m}^{3}$ (specific illuminated surface area $80 \mathrm{~m}^{-1}$, depth of culture $0.013 \mathrm{~m}$ ). The flat panel part was made of stainless steel except for the illuminated front surface, which was made of glass. The solution was mixed by pumping with a re-circulation fluid loop, a glass tube, placed on the side of the PBR. The solution input was at the bottom of the reactor. A volumetric pump delivering a constant flow rate of $2.5 \times 10^{-5} \mathrm{~m}^{3} \mathrm{~s}^{-1}$ was used, corresponding to a fully developed laminar flow $(\operatorname{Re}=160)$. The PBR was slightly inclined with a tilt angle of $3^{\circ}$. We note that the high specific illuminated surface of such a system could be expected to offer a high volumetric biomass productivity, but this point lies outside the scope of this study.

Optimization of a solar PBR production is complex, because of the high dependency of the process behaviour on solar radiation, and of the possibility of applying various harvesting strategies to optimize light attenuation conditions inside the culture bulk, which greatly influence PBR productivity [10]. A general rule is that best biomass productivity is obtained with complete absorption of the incident photons flux density (termed light-limited conditions) $[25,26]$. To meet these conditions, experiments were performed with water to which a black dye was added. Although such a solution is not fully representative of a microalgae culture (in terms of optical properties), absorption is very close, as most of the

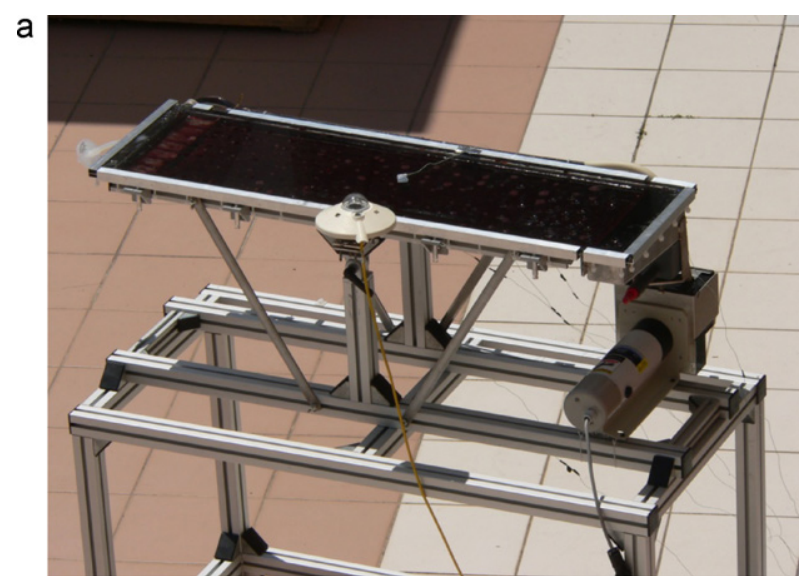

b

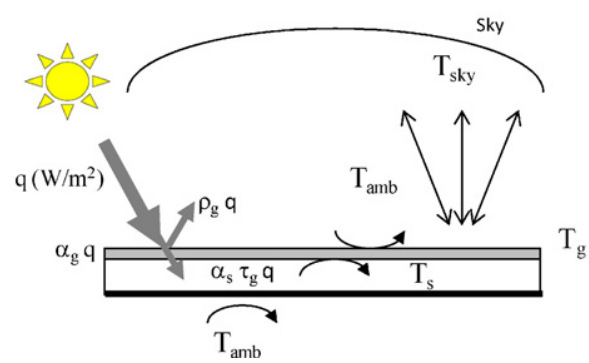

Fig. 1. (a) The flat panel reactor during outdoor thermal tests. (b) Schematic drawing of the modeling with the solar radiation $(\longrightarrow)$, the heat exchange by convection $(\longrightarrow$ ) and radiation $(\leftrightarrow)$ between the PBR made up of a glass ( $\square$ ), the solution ( $\square$ ) and the underside ( $)$. The underside of the reactor and the solution are assumed to be at the same temperature $\left(T_{S}=T_{u}\right)$.

irradiation spectrum is absorbed in light-limited conditions by cell pigments or by the water itself.

The reactor was instrumented to collect the data necessary for the thermal analysis. Temperature was monitored with a set of acquisition boards (National instrument USB-6009) and software (LabVIEW), and probes (thermocouples type K) were set at different locations in the system (in the solution, on the optical glass surface, on the underside of the steel reactor and in the ambient air). The global (or total) light flux $q$ was measured with a pyranometer (Kipp and Zonen CMP3) placed in the plane of the reactor and covering the spectral range $300-2800 \mathrm{~nm}$.

\section{Indoor experiments}

A set of indoor experiments was performed with artificial illumination of the glass surface in an air-conditioned room. The uniform illumination $\left( \pm 6 \%\right.$ ) was provided by a LED panel of area $1 \mathrm{~m}^{2}$ producing light in the visible spectral range $0.4-0.7 \mu \mathrm{m}$, also termed photosynthetically active radiation (PAR). Radiation was controlled and could be delivered at irradiance levels of a few tens of Watts per square meter to $500 \mathrm{~W} \mathrm{~m}^{-2}$, the maximum value of the visible part of solar radiation. Indoor experiments offer the advantage of allowing accurate control of the environmental conditions: both ambient temperature and irradiation can be easily regulated.

Firstly, the reactor underwent successive irradiation steps until thermal equilibria were reached (Fig. 2). Secondly, transient operating conditions were imposed on the reactor with an irradiation profile (in the PAR range) representative of a sunny day (Fig. 3). The objective of these experiments was to perform a first validation of the thermal model developed in the next section by comparison of experimental and simulated temperature profiles. A first insight was also obtained into PBR thermal behaviour. The temperature at the underside of the reactor was found to be very close to the 

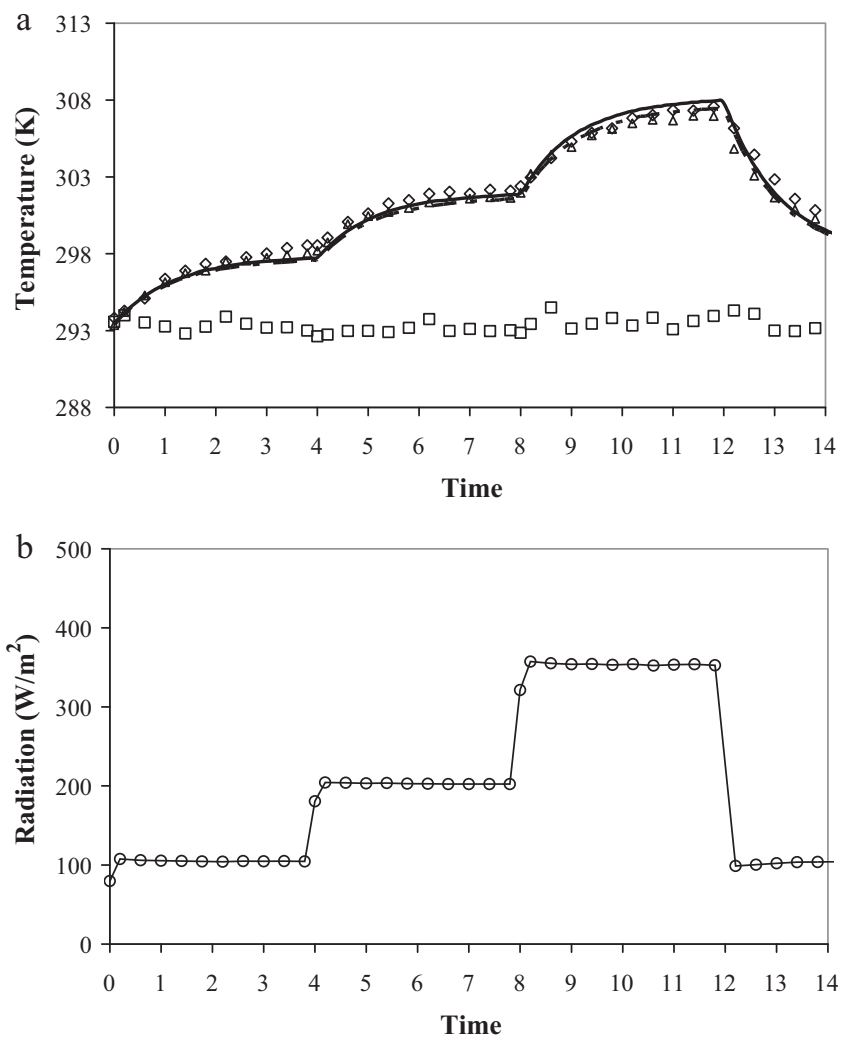

Fig. 2. (a) Experimental $((\Delta)=$ glass; $(\diamond)=$ solution; $(\square)=$ ambient) and simulated (dashed line = glass; continuous line $=$ solution $)$ temperature profiles during successive radiation gaps. (b) Radiation steps in the reactor.
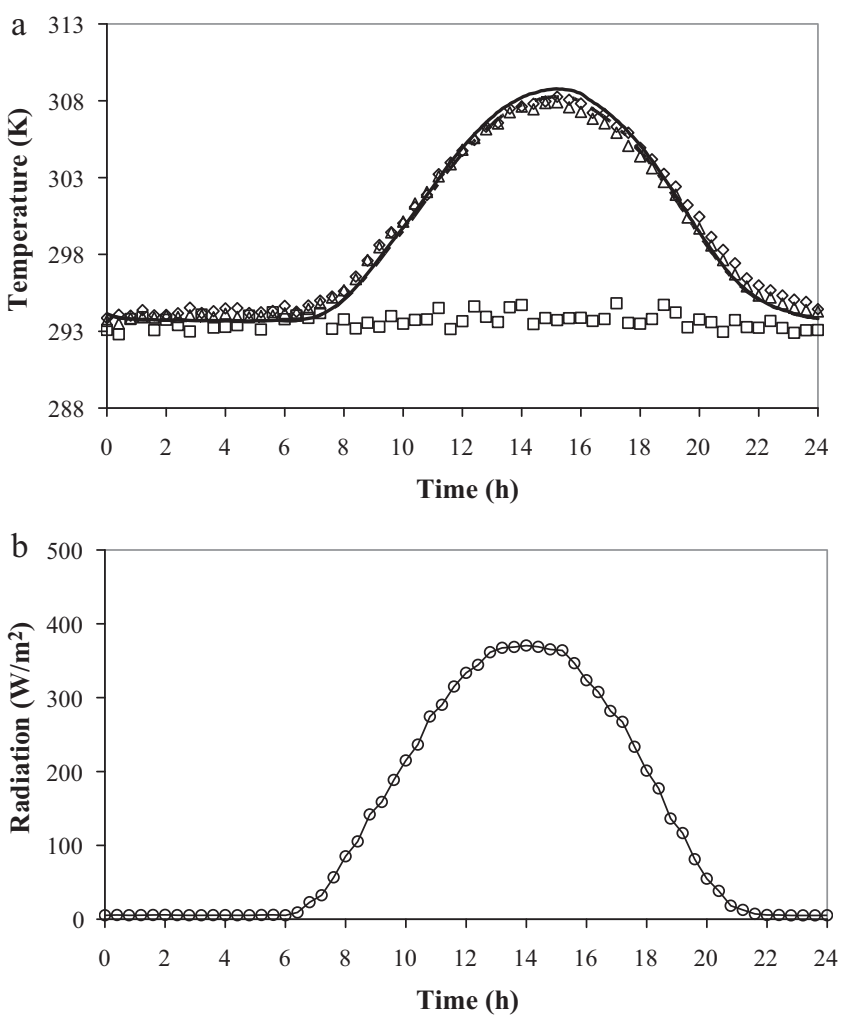

Fig. 3. (a) Indoor experimental $((\Delta)=$ glass; $(\diamond)=$ solution; $(\square)=$ ambient) and simulated (dashed line=glass; continuous line =solution) temperature profiles. $(b)$ Indoor radiation profile.
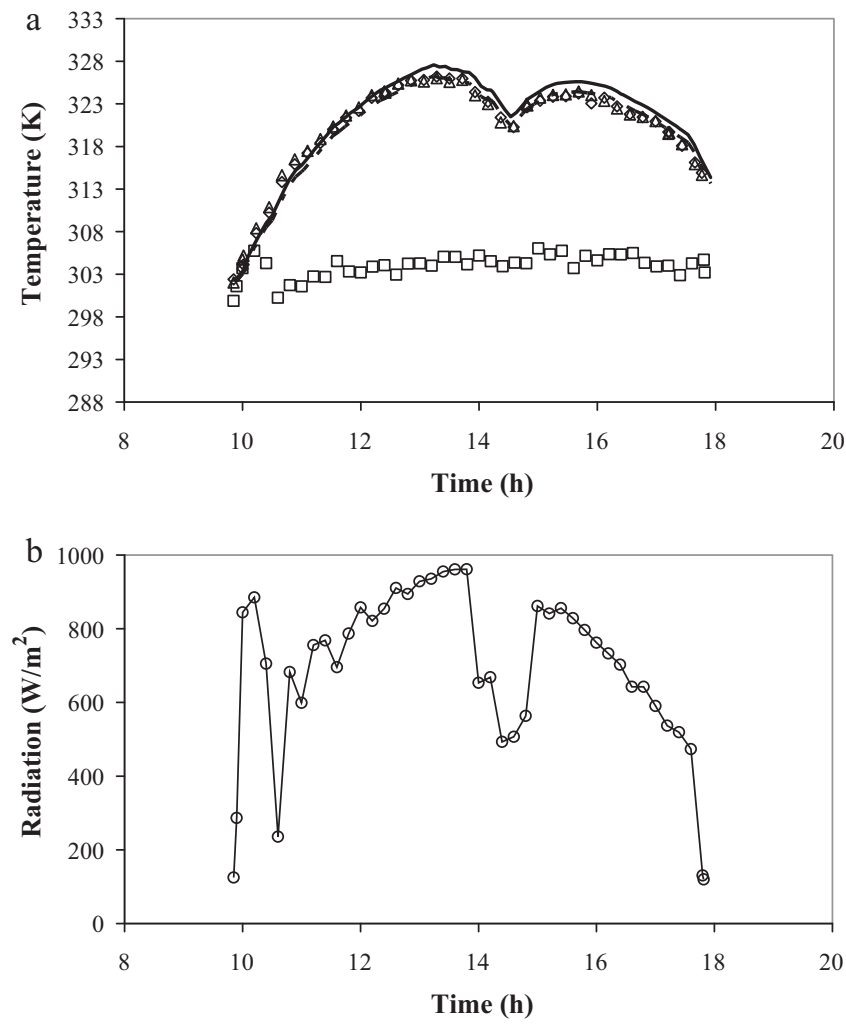

Fig. 4. (a) Outdoor experimental $((\Delta)=$ glass; $(\diamond)=$ solution; $(\square)=$ ambient ) and simulated (dashed line=glass; continuous line=solution) temperature profiles. (b) Outdoor radiation profile.

solution temperature, and is not represented in the figure. Similarly, the temperature of the glass was almost equal to the temperature of the solution. From a thermal point of view, we observed that the system could be considered as being at a uniform temperature. This is accounted by the reactor sides, which transfer heat between the solution and the ambient air. Because heat exchange in forced convection between a liquid and a surface is much higher than heat exchange by natural convection between the air and the same surface, all the surfaces in contact with the circulating culture will have almost the same temperature.

\section{Outdoor experiments}

Outdoor experiments were performed in summer at Perpignan in the south of France. Fig. 4 shows the temperature and the solar radiation profiles recorded for a typical outdoor experiment. On this day, solar radiation reached a peak close to $1000 \mathrm{~W} \mathrm{~m}^{-2}$. The cloudy period in the middle of the day revealed the low thermal inertia of the system. The temperatures decreased as soon as the light flux decreased. For two hours, between 1 p.m. and 3 p.m., the mean solar radiation of $750 \mathrm{~W} \mathrm{~m}^{-2}$ entailed a mean temperature of the solution of $323 \mathrm{~K}$. The maximum temperature of the solution, $328 \mathrm{~K}$, was too high to be endured by most of the photosynthetic microorganisms without serious damage.

\section{Thermal modeling}

Although the system can be considered to be at an even temperature, it was modeled (to check the homogeneity of the simulated system) as two distinct sub-systems: a first sub-system formed of the upper glass, and a second sub-system comprising the solution and the underside of the reactor, assumed to be at the same temperature (Fig. 1b). 
The thermal balance considering convection and radiation heat transfer yields the following equations for the outdoor experiments:

$$
\begin{aligned}
& m_{\mathrm{g}} C p_{\mathrm{g}} \frac{\mathrm{d} T_{\mathrm{g}}}{\mathrm{d} t}=q S_{\mathrm{g}} \alpha_{\mathrm{g}}-\varepsilon_{\mathrm{g}} \sigma S_{\mathrm{g}}\left(T_{\mathrm{g}}{ }^{4}-T_{\mathrm{sky}}{ }^{4}\right)-h_{\mathrm{g} / \mathrm{s}} S_{\mathrm{g}}\left(T_{\mathrm{g}}-T_{\mathrm{s}}\right) \\
& \quad-h_{\mathrm{g} / \mathrm{amb}} S_{\mathrm{g}}\left(T_{\mathrm{g}}-T_{\mathrm{amb}}\right) \\
& \left(m_{\mathrm{s}} C p_{\mathrm{s}}+m_{\mathrm{u}} C p_{\mathrm{u}}\right) \frac{\mathrm{d} T_{\mathrm{s}}}{\mathrm{d} t}=q S_{\mathrm{s}} \alpha_{\mathrm{s}} \tau_{\mathrm{g}}-h_{\mathrm{g} / \mathrm{s}} S_{\mathrm{s}}\left(T_{\mathrm{s}}-T_{\mathrm{g}}\right) \\
& \quad-h_{\mathrm{u} / \mathrm{amb}} S_{\mathrm{u}}\left(T_{\mathrm{s}}-T_{\mathrm{amb}}\right)
\end{aligned}
$$

with $T_{\mathrm{g}}$, and $T_{\mathrm{S}}$ respectively the glass and solution temperatures. The ambient temperature $T_{\mathrm{amb}}$ and the total solar radiation $q$ at the glass surface $S_{\mathrm{g}}$ are input data recorded during the experiments; $q$, the total radiation measured by the pyranometer, is the sum of the direct and diffuse solar radiations. The optical properties of the glass and solution are given in Table 1 with the heat capacities and the masses of the components involved.

For the outdoor experiments, the heat exchange by radiation between the external underside of the reactor and the ground is neglected (assuming the ground under irradiation to be at a temperature close to that of the underside). Radiations due to atmospheric emission are expressed by an equivalent sky temperature [27]:

$T_{\text {sky }}=0.0552 T_{\mathrm{amb}} 1.5$

The convection heat exchange coefficient between the two subsystems (glass and solution) is $h_{\mathrm{g} / \mathrm{s}}=290 \mathrm{~W} / \mathrm{m}^{2} \mathrm{~K}$. It was calculated with a Nusselt number equal to 8.23 , corresponding to a fully developed laminar flow $(\mathrm{Re}=160)$ in a pipe of rectangular cross section with a very high geometry shape factor (ratio of the two sides) [28]. The only unknown parameter needed to solve the set of ordinary differential equations was the convection heat exchange coefficient $h_{\mathrm{g} / \mathrm{amb}}=h_{\mathrm{u} / \mathrm{amb}}$ between the ambient air and the closed system. Its value can be approached by the empirical correlation [29]:

$h_{\mathrm{g} / \mathrm{amb}}=5.7+3.8 v$

with $v$ the wind speed in $\mathrm{m} \mathrm{s}^{-1}$.

The thermal model does not take into consideration the flow of $\mathrm{CO}_{2}-$ air-enriched necessary to ensure light limited growth in the production of microalgae. From a thermal point of view, this flow will probably change the absolute value of the heat exchange coefficient $h_{\mathrm{g} / \mathrm{s}}$ between the glass and the solution. But such exchange is nevertheless not limiting in comparison with the main convective heat transfer limitation that is the result of the heat exchange between the ambient air and the glass. Aeration contributes also to evaporation that will happen in the PBR, producing a cooling effect. Nevertheless: (i) taking as reference the air mass flow equal to $1.1 \times 10^{-6} \mathrm{~kg} \mathrm{~s}^{-1} \mathrm{~m}^{-2}$ selected by Hu et al. [30] for their experiments in a tilted and closed PBR, the corresponding air flow for the reactor tested in this study would be equal to $0.36 \times 10^{-6} \mathrm{~kg} \mathrm{~s}^{-1}$; (ii) considering an inlet air flow at a humidity rate of $60 \%$ and an inlet temperature of $298 \mathrm{~K}$; (iii) a $\mathrm{CO}_{2}$ air-enriched flowing at the outlet of the reactor at saturation; the mass flow of water evaporated would be equal to $0.17 \times 10^{-7} \mathrm{~kg} \mathrm{~s}^{-1}$, corresponding to a cooling power lower than $0.05 \mathrm{~W}$. The contribution on the heat balance on the process could thus be neglected. This is here explained by the low water evaporation that is one of the advantages of closed
PBRs. Obviously, such conclusion is not valid to shallow algal ponds where evaporation is important [31].

In indoor experiments, the room has to be considered as a cavity with a wall at uniform constant temperature equal to the ambient temperature, $294 \mathrm{~K}$ (Fig. 3). Black body radiation exists in a cavity irrespective of whether the cavity surface is highly reflecting or absorbing [28]. $T_{\mathrm{amb}}$ is then substituted for $T_{\text {sky }}$ in Eq. (1) and the term for radiation exchange between the ground, considered as a black body (at $294 \mathrm{~K}$ ), and the reactor underside is added.

For indoor conditions, the wind speed was taken to be equal to 0 . For outdoor conditions, the best fit was obtained for a value of the heat exchange coefficient $h_{\mathrm{g} / \mathrm{amb}}$ of $11 \mathrm{~W} / \mathrm{m}^{2} \mathrm{~K}$. This corresponds to a low wind speed $\left(1.5 \mathrm{~m} \mathrm{~s}^{-1}\right)$ consistent with the fact that outdoor experiments were carried out in a courtyard protected from wind.

\section{Thermal model validation}

For indoor experiments (Figs. 2 and 3), all the values necessary for the resolution of the ordinary differential equations system are known before simulation (no fitting): (i) the measurements of the lamp radiations and the ambient temperatures; (ii) the data derived from the design of the PBR (surfaces, masses, ...), the materials (glass, steel, water, ... ) involved and their thermal and optical properties (Table 1 ). The agreement between simulated and experimental temperature profiles (without any parameter identified or adjusted) for the two kinds of experiments: successive radiations gaps; radiations profiles "representative" of a sunny day; can be considered as a validation of the thermal model. For radiations gaps, the mean relative error between the simulated and the experimental temperatures of the glass and the solution is equal to $1.4 \times 10^{-3}$. For the sunny day representative radiation profile, this error is of $1.6 \times 10^{-3}$. The maximum temperature reached by the solution during the outside experiment was correctly estimated, as well as the transient regime induced by the cloudy period in the middle of the day for the outdoor experiment. For the one day of operation, the mean relative error between the simulated and the experimental temperature profiles reaches $5.8 \times 10^{-3}$.

All these results tend to validate the model developed here in the particular case of a flat panel solar PBR with a high ratio of illuminated surface area to volume, and so with a low thermal inertia.

\section{Simulations of annual temperature profiles}

Issues related to the PBR temperature control of outdoor PBR can be especially highlighted by simulating thermal behaviour during a full working year. The thermal model was thus used for this purpose. The yearly meteorological data necessary were generated with Meteonorm software (www.meteonorm.com) based on tenyear measurement periods.

Simulations were conducted for the reactor described in the experimental section and located at Perpignan $\left(42.2^{\circ} \mathrm{N}, 2.54^{\circ} \mathrm{W}\right)$. For this area of the south of France, the total amount of the solar resource available and the annual mean ambient temperature are respectively $1440 \mathrm{kWh} \mathrm{m}^{-2}$ and $283 \mathrm{~K}$. This is also an area with a very windy period and a mean wind speed of $5 \mathrm{~m} \mathrm{~s}^{-1}$. An example of the temperature profiles during one week in summer is pre-

\begin{tabular}{|c|c|c|c|c|c|c|}
\hline Component & $m(\mathrm{~kg})$ & $C p(\mathrm{~J} / \mathrm{kg} \mathrm{K})$ & $S\left(\mathrm{~m}^{2}\right)$ & $\alpha(-)$ & $\varepsilon(-)$ & $\tau(-)$ \\
\hline Glass & 2.6 & 720 & 0.33 & 0.05 & 0.92 & 0.90 \\
\hline Solution, water and black dye & 0.65 & 4180 & 0.33 & 1 & 1 & - \\
\hline Underside of reactor & 12 & 500 & 0.5 & 0.58 & 0.58 & - \\
\hline
\end{tabular}
sented in Fig. 5. As expected, and in agreement with the previous

Table 1

Constants and data used in the thermal balance. 

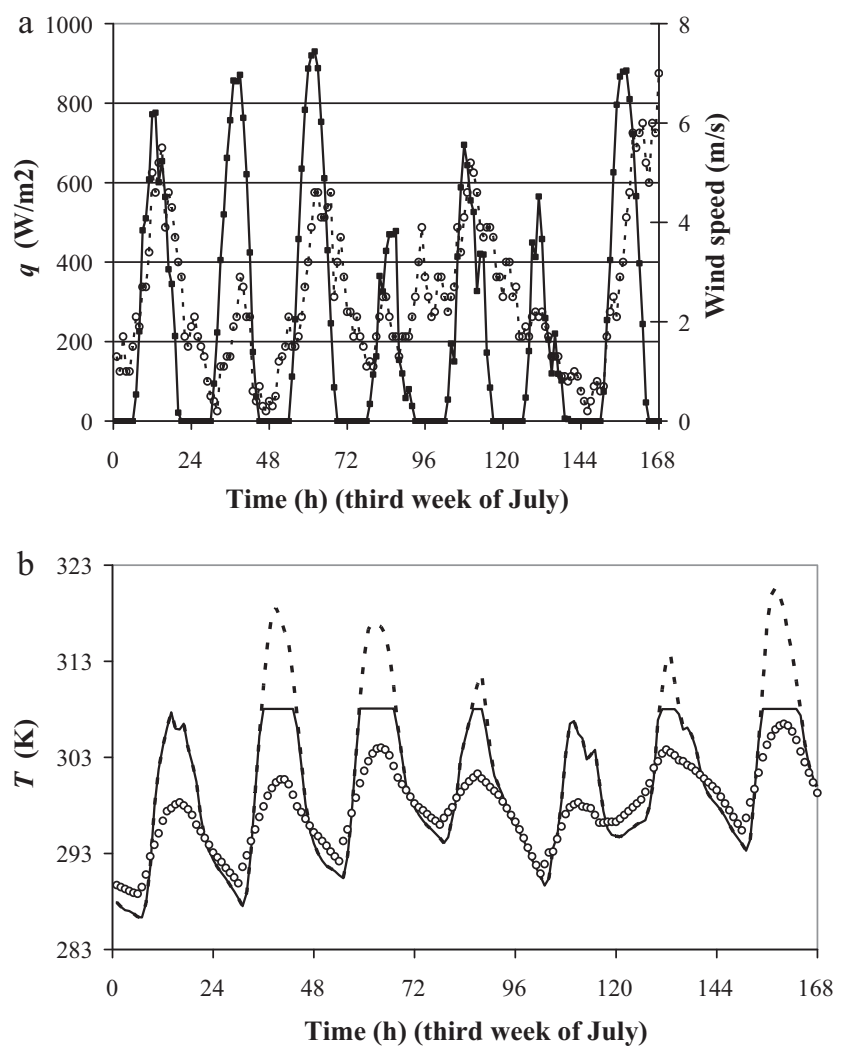

Fig. 5. (a) Total irradiation ( $\longrightarrow-$ ) and wind speed $(-\infty-\cdots)$ during the third week of July (provided by Meteonorm). (b) Ambient temperature $(\bigcirc)$ and simulated culture temperature with an external temperature control system (continuous line) and with no temperature control (dashed line).

experimental results, during the sunny days when the global radiation values are close to $900 \mathrm{~W} \mathrm{~m}^{-2}$ (Fig. 5a), the simulated temperatures of the solution (Fig. 5b) exceed values that can be tolerated by microorganisms. The year-round simulation reveals that this problem is not limited to the sunniest days in summer (Fig. 6) and is encountered during almost half the year. From May to September, a temperature higher than $323 \mathrm{~K}$ is reached at least for one hour per month in the culture. Such a temperature would obviously be lethal for photosynthetic microorganisms and must therefore be avoided in microalgal cultivation.

By means of external cooling, it is possible to maintain the system below a preset temperature ceiling. Basically, the cooling

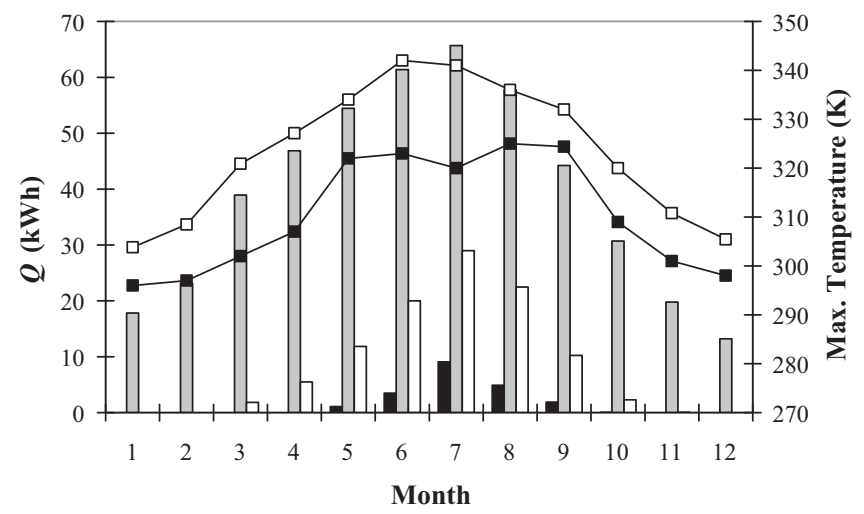

Fig. 6. Site of Perpignan. Histogram of the irradiation received by the reactor (grey), the heat to be extracted to keep the temperature below $308 \mathrm{~K}$ with data provided by Meteonorm (black) and with the same data except for the wind speed taken equal to 0 (white). Maximum temperatures with $(-\mathbf{\square}$ ) and without wind ( $\square-\square)$. system (heat exchange fluid loop or water spraying at the surface of the reactor) is activated as soon as the culture temperature exceeds the preset value. This can be simulated by adding a heat sink term in the thermal balance of the culture (Eq. (1)). This will allow estimation at each time step of the instantaneous power that must be extracted to avert an increase in the temperature above the ceiling. The corresponding energy can then be calculated by integration of the power extracted during the time step.

A preset ceiling of $308 \mathrm{~K}$ was specified. This value was chosen as representative of the maximum temperature tolerated by different types of microorganism without serious damage [11]. Obviously, any other preset temperature could be simulated using the thermal model. Fig. 5b shows the temperature profile obtained with thermal regulation. It was found necessary to cool the system to avoid overheating for a total time of $370 \mathrm{~h}$, mainly in summer. The total amount of energy to be extracted is thus $21 \mathrm{kWh}$ for the whole year. This represents $4.5 \%$ of the solar energy received on the glass illuminated surface (Fig. 6). In the first instance, such a value could be considered negligible. However, for specific applications such as biofuel production, the energy balance is of crucial importance and must be positive. The maximum energy yield of sunlight conversion into biomass is around $15-18 \%$ in the photosynthetically active radiation bandwidth (6.5-7.5\% of the total sunlight spectrum). In the practical case of a PBR with direct solar illumination, values of around $5 \%$ ( $2 \%$ of the total spectrum) are obtained [32]. The energy used for thermal regulation was thus found here to be of the same order of magnitude as that recovered in biomass. Considering that thermal regulation is not the only cause of energy consumption in the overall process, the utility of producing algae for biofuel purposes using the system studied here is clearly greatly reduced.

Additional simulations were conducted here to study relevant parameters affecting the thermal balance of a closed solar PBR. We found a high sensitivity to the heat exchange coefficients, correlated here to the wind conditions (Eq. (4)). These conditions range widely according to the local sitting of the reactor. An interesting extreme working condition for this process is given by a reactor installed in a wind-protected area $(v=0)$. In this case, more than $20 \%$ of the solar energy received by the system during one year of operation has to be evacuated, and $40 \%$ in the month of July (Fig. 6). Again, this underlines the need to take into consideration the energy cost of the thermal regulation. The marked difference between the results obtained with and without wind emphasizes, as it is always the case in the use of a renewable energy, the importance of the choice of the local site for the production of microorganisms with closed PBRs. Obviously, the results are also highly sensitive to the level of the maximum temperature tolerated by the microorganisms, as well as the geographical area considered. Consequently the values determined with the 1-year simulation (number of hours above $308 \mathrm{~K}$, total amount of energy necessarily to extract) are not to be considered as absolute and universal values. They only tend to demonstrate that in most of the cases of closed PBRs implemented in area with high levels of solar radiation and high ambient temperature, it will be probably necessary to take thermal management into consideration to optimize the productivity.

A PBR can also be engineered to reduce its energy consumption for thermal regulation. In the solar energy spectrum, only the visible part of the radiation is useful for photosynthetic mechanisms. The ultraviolet part, which represents 5\% (AM1.5 Spectrum, Norm ASTM) of the solar energy spectrum at the Earth's surface, is filtered by the glass optical surface. An efficient way to limit the temperature increase of the solution during sunny days would be to use filter glass to limit the input of the solar infrared radiation (IR) to the culture bulk. Reflection of IR wavelengths impinging the PBR surface, almost 55\% of the total solar radiation, is possible using special glass such as hot mirrors designed to transmit visible wavelengths while reflecting and/or absorbing near-infrared, 
Table 2

Maximum temperatures of the culture and time at temperatures higher than $308 \mathrm{~K}$ as a function of reflectivity $\rho(\alpha=0.05)$.

\begin{tabular}{lcccccl}
\hline$\rho$ & 0.05 & 0.1 & 0.2 & 0.3 & 0.4 & 0.5 \\
\hline$T_{\max }$ & 52.3 & 50.9 & 48.16 & 45.4 & 42.6 & 39.9 \\
Time (h) & 367 & 315 & 246 & 182 & 122 & 63 \\
\hline
\end{tabular}

heat-generating wavelengths. This option is particularly attractive since it needs no regulation or external cooling system (passive system). The thermal model can be easily used to simulate this approach by modifying the optical properties of the filter glass. For example, increasing the absorbance of the glass to decrease the amount of radiation reaching the culture is ineffectual: with $90 \%$ of the IR radiation absorbed ( $\tau=0.45$ and $\alpha=0.5$ for the total solar radiation) a peak culture temperature of $325 \mathrm{~K}$ is reached in August, very close to that obtained with ordinary glass. This is because the solar energy absorbed by the upper glass surface is mostly transferred to the culture and contributes to its heating. The reflective properties of the illuminated surface thus appear here to be a more relevant parameter (Table 2 ). For optical hot mirrors with $90 \%$ of the IR radiation reflected ( $\tau=0.45$ and $\alpha=0.10$ for the total solar radiation), simulation of temperature profiles and thermal balance gives: (i) a maximum temperature of the culture lower than $313 \mathrm{~K}$ in August, (ii) only 63 working hours during the whole year with the culture above the temperature limit of $308 \mathrm{~K}$ (against $370 \mathrm{~h}$ with standard glass) and (iii) less than $0.5 \%$ of the solar energy collected over a year to be extracted to keep below the temperature ceiling (4.5\% with standard glass).

\section{Conclusion}

High productivity implies maximizing capture of solar radiation, but due to the high flux of sunlight, the culture can overheat. A thermal model for closed solar PBR was proposed and validated. This model combined with meteorological data was firstly used to simulate the year-round thermal time course of the culture bulk. It was found that a reactor implemented in an area with climate conditions such as in the south of France, is likely to reach excessive temperature (upper $308 \mathrm{~K}$ ) during several hundred of hours. This could be criticism for the productivity of the reactor because overheating is lethal for living cells and has to be avoided throughout the production period (ideally all year). So, excepted the case where a heat sink is available near the local site of production (e.g. sea water), thermal regulation can become a critical point in the specific context of solar closed PBRs. Management of the temperature profiles of a closed PBR implies robust and optimized regulation procedures. A thermal model able to catch the transient behaviour of a solar PBR is useful in this context, for example, to set advanced thermal control strategies integrating PBR thermal inertia and radiation dynamics (including night influence) for a given location, period of production and PBR geometry. As an illustrative example, a simple regulation with a ceiling temperature was tested in this work. It was found in particular that the energy consumption for thermal regulation could reach that recovered in biomass, emphasizing its critical relevance in biofuel production, where a positive energy balance is of importance. Obviously, there is also a lower temperature threshold, and appropriate temperature control may thus also be necessary, especially in winter because of low ambient temperatures and sun radiation. Thermal regulation would be necessary at least to prevent the culture freezing. Low temperature also influences the kinetic growth.

Microorganisms grow within a narrow temperature band, with a non-linear influence of temperature on growth. It would thus be interesting to associate a kinetic model of photosynthetic growth including both temperature and light influences with the thermal model and to an already available PBR model able to represent light-limited growth in solar conditions. This could be used for an overall optimization of a solar PBR by considering the two main issues of outdoor production, namely radiation capture and thermal regulation. As major results of such a tool, it could help in the PBR engineering but also to investigate PBR efficiency as a function of its location, in terms of both, biomass productivity and energy consumption.

\section{Acknowledgements}

This work was supported by the French National Research Agency for Bioenergy Production (ANR-PNRB), and is part of the French "Biosolis" research program on developing photobioreactor technologies for mass-scale solar production (http://www.biosolis.org/)

\section{References}

[1] M.L. Ghirardi, L. Zhang, J.W. Lee, T. Timothy Flynn, S. Seibert, E. Greenbaum, A. Melis, Microalgae: a green source of renewable $\mathrm{H}_{2}$, Trends Biotechnol. 18 (2000) 506-511.

[2] A. Melis, Green alga hydrogen production: progress, challenges and prospects, Int. J. Hydrogen Energy 27 (2002) 1217-1228.

[3] A.H. Scragg, A.M. Illman, A. Carden, S.W. Shales, Growth of microalgae with increased calorific values in a tubular bioreactor, Biomass Bioenergy 23 (2002) 67-73.

[4] J.R. Benemann, Hydrogen and methane production by microalgae, in: Handbook of Microalgal Culture: Biotechnology and Applied Phycology, Blackwell Science Ltd., Iowa, 2004.

[5] P. Spolaore, C. Joannis-Cassan, E. Duran, A. Isambert, Commercial applications of microalgae, J. Biosci. Bioeng. 101 (2006) 87-96.

[6] Y. Chisti, Biodiesel from microalgae, Biotechnol. Adv. 25 (2007) 294-306.

[7] A.L. Ahmad, N.H.M. Yasin, C.J.C. Derek, J.K. Lim, Microalgae as a sustainable energy source for biodiesel production: a review, Renew. Sustain. Energy Rev. 15 (2010) 584-593.

[8] A.P. Carvalho, L.A. Meireles, F.X. Malcata, Microalgal reactors: a review of enclosed system designs and performances, Biotechnol. Prog. 22 (2006) 1490-1506.

[9] F. Lehr, C. Posten, Closed photo-bioreactors as tools for biofuel production, Curr. Opin. Biotechnol. 20 (2009) 280-285.

[10] J. Pruvost, J.F. Cornet, V. Goetz, J. Legrand, Modeling dynamic functioning of rectangular photobioreactors in solar conditions, AIChEJ. 57 (2011) 1947-1960.

[11] A. Richmond, Principles for attaining maximal microalgal productivity in photobioreactors: an overview, Hydrobiologia 512 (2004) 33-37.

[12] C.U. Ugwu, H. Aoyagia, H. Uchiyamaa, Photobioreactors for mass cultivation of algae, Bioresour. Technol. 99 (2008) 4021-4028.

[13] H.U. Qiang, Y. Zarmi, A. Richmond, Combined effects of light intensity, lightpath and culture density on output rate of Spirulina platensis (Cyanobacteria), European J. Phycol. 33 (1998) 165-171.

[14] J. Doucha, K. Lívanský, Productivity, $\mathrm{CO}_{2} / \mathrm{O}_{2}$ exchange and hydraulics in outdoor open high density microalgal (Chlorella sp.) photobioreactors operated in a Middle and Southern European climate, J. Appl. Phycol. 18 (2006) 811-826.

[15] H. Qiang, D. Faiman, A. Richmond, Optimal tilt angles of enclosed reactors for growing photoautotrophic microorganisms outdoors, J. Ferment. Bioeng. 85 (1998) 230-236

[16] A. Converti, A.A. Casazza, E.Y. Ortiz, P. Perego, M. Del Borghi, Effect of temperature and nitrogen concentration on the growth and lipid content of Nannochloropsis oculata and Chlorella vulgaris for biodiesel production, Chem. Eng. Proc.: Process Intensif. 48 (2009) 1146-1151.

[17] G. Torzillo, A. Sacchi, R. Materassi, Temperature as an important factor affecting productivity and night biomass loss in Spirulina platensis grown outdoors in tubular photobioreactors, Bioresour. Technol. 38 (1991) 95-100.

[18] I. Rafiqul, A. Hassan, G. Sulebele, C. Orosco, P. Roustaia, Influence of temperature on growth and biochemical composition of Spirulina platensis and S. fusiformis, Iranian Int. J. Sci. 4 (2003) 97-106.

[19] A. Vonshak, Spirulina platensis (Arthrospira): Physiology, Cell-biology, and Biotechnology, Taylor \& Francis, 1997.

[20] P. Carlozzi, A. Sacchi, Biomass production and studies on Rhodopseudomonas palustris grown in an outdoor, temperature controlled, underwater tubular photobioreactor, J. Biotechnol. 88 (2001) 239-249.

[21] E. Molina, J. Fernandez, F.G. Acien, Y. Chisti, Tubular photobioreactor design for algal cultures, J. Biotechnol. 92 (2001) 113-131.

[22] Z. Cheng-Wu, O. Zmora, R. Kopel, A. Richmond, An industrial-size flat plate glass reactor for mass production of Nannochloropsis sp. (Eustigmatophyceae) Aquaculture 195 (2001) 35-49.

[23] G. Chini Zittelli, F. Lavista, A. Bastianini, L. Rodolfi, M. Vincenzini, M.R. Tredici, Production of eicosapentaenoic acid by Nannochloropsis sp. cultures in outdoor tubular photobioreactors, J. Biotechnol. 70 (1999) 299-312. 
[24] G. Torzillo, B. Pushparaj, F. Bocci, W. Balloni, R. Materassi, G. Florenzano, Production of Spirulina biomass in closed photobioreactors, Biomass 11 (1986) 61-74.

[25] J.F. Cornet, C.G. Dussap, A simple and reliable formula for assessment of max imum volumetric productivities in photobioreactors, Biotechnol. Progr. 25 (2009) 424-435.

[26] H. Takache, G. Christophe, J.F. Cornet, J. Pruvost, Experimental and theoretical assessment of maximum productivities for the micro-algae Chlamydomonas reinhardtii in two different geometries of photobioreactors, Biotechnol. Progr. 26 (2010) 431-440.

[27] J.A. Duffie, W.A. Beckman, Solar Engineering of Thermal Processes, third ed. John Willey \& Sons, New York, 2006.
[28] F.P. Incoprera, D.P. DeWitt (Eds.), Fundamentals of Heat and Mass Transfer, fourth ed., John Willey \& Sons, New York, 1996.

[29] W.H. McAdams, Heat Transmission, third ed., Mc Graw-Hill, New York, 1954.

[30] Q. Hu, H. Guterman, A. Richmond, A flat inclined modular photobioreactor for outdoor mass cultivation of photoautotrophs, Biotechnol. Bioeng. 51 (1996) 51-60.

[31] Q. Béchet, A. Shilton, J.B.K. Park, R.J. Craggs, B. Guieysse, Universal temperature model for shallow algal ponds provides improved accuracy, Environ. Sci. Technol. 45 (2011) 3702-3709.

[32] J.F. Cornet, Calculation of optimal design and ideal productivities of volumetrically-lightened photobioreactors using the constructal approach, Chem. Eng. Sci. 65 (2010) 985-998. 